\title{
Testing Newton/GR, MoND and quantised inertia on wide binaries
}

\author{
M.E. McCulloch ${ }^{1}$ (D) J.H. Lucio ${ }^{1}$
}

Received: 6 March 2019 / Accepted: 27 July 2019 / Published online: 5 August 2019

(c) The Author(s) 2019

\begin{abstract}
Wide binary stars are within the low-acceleration regime in which galactic rotation curves deviate from Newtonian or general relativistic predictions. It has recently been observed that their rotation rates are similarly anomalous in a way that dark matter cannot explain, since it must be smooth on these small scales to fit galaxy rotation curves. Here, it is shown that Newtonian/GR models cannot predict these wide binaries since dark matter cannot be applied. It is also shown that MoND cannot predict these systems. However, a model which assumes that inertia is due to Unruh radiation made inhomogeneous in space by relativistic horizons (QI, quantised inertia) can predict these wide binaries, and it has the advantage of not needing an adjustable parameter.
\end{abstract}

Keywords Wide binaries quantised inertia MoND

\section{Introduction}

Zwicky (1933) first noticed that the motion of galaxy clusters was too energetic to be held together by visible matter, assuming Newtonian or general relativistic physics, and proposed the existence of an invisible (dark) matter that provides the extra required gravitational pull. A similar problem in disc galaxy rotation was proven by the higher quality galaxy rotation curves obtained by Rubin et al. (1980). Dark matter is still the most popular explanation for the galaxy rotation problem, but, after decades of searching, dark matter has not been directly detected, though many efforts are ongoing, such as Ahmed et al. (2009) and the XENON10 Collaboration (2010).

M.E. McCulloch

mike.mcculloch@plymouth.ac.uk

1 University of Plymouth, PL4 8AA, Plymouth, UK
Milgrom (1983) proposed an alternative explanation for galaxy rotation. He speculated that either (1) the force of gravity increases or (2) the inertial mass $\left(M_{\mathrm{I}}\right)$ decreases for the low accelerations at a galaxy's edge. His empirical scheme, called Modified Newtonian Dynamics (MoND), can fit galaxy rotation curves and has the advantage of being less tunable than dark matter. However, it does require tuning by one arbitrary parameter, the acceleration $a_{0}$, it does not suggest a specific mechanism and it does not predict the dynamics of galaxy clusters.

A theory has been proposed, see McCulloch (2007, 2013, 2016), in which inertia arises solely from a push on objects by the quantum vacuum, which is made more intense by acceleration (Unruh radiation) and made non-uniform in space by relativistic acceleration-dependent Rindler horizons and able to push on matter. The theory predicts galaxy rotation without dark matter and without any adjustment, see McCulloch (2012, 2017a), and it implies that it is possible to produce new dynamics by artificially creating horizons, damping the quantum vacuum, making it inhomogeneous and able to push on objects, see McCulloch (2017b). In QI the inertial mass becomes

$M_{\mathrm{I}}=M_{\mathrm{g}}\left(1-\frac{2 c^{2}}{A \Theta}\right)$

where $m_{\mathrm{g}}$ is the gravitational mass, $c$ is the speed of light, $A$ is the total acceleration of the object relative to the fixed stars, and $\Theta$ is the distance to the co-moving cosmic diameter, $8.8 \times 10^{26} \mathrm{~m}$. This is a generally-accepted estimate of the cosmic diameter assuming that inflation has pushed objects beyond the distance that we can now see (see Bars and Terning 2009). This represents the diameter as it is now and not when the light was emitted from the horizon. For the derivation of Eq. (1) see McCulloch (2007, 2016). QI successfully 
predicts galaxy rotation without dark matter, see $\mathrm{McCul}-$ loch $(2012,2017 \mathrm{a})$, and the interesting pattern noticed by Sanders and McGaugh (2002) that the anomalous behaviour in galaxies begins at the radius where the acceleration of the stars drops below the acceleration of $a_{0} \sim 2 \times 10^{-10} \mathrm{~m} / \mathrm{s}^{2}$. The problem is that galaxies do not provide a clean test since dark matter can be 'fitted' to also explain them.

The much simpler globular clusters were studied by Scarpa et al. (2007), who observed the same change in behaviour at the critical acceleration. Hernandez (2012), and later with better GAIA DR2 (Data Release 2) data, Hernandez (2019), provided a brilliantly simple crucial experiment: they looked at the behaviour of wide-orbit binary stars (for which the critical acceleration $a_{0}$ occurs at a separation of about $7000 \mathrm{AU}$ or $0.03 \mathrm{pc}$ ). Again, they found that the start of anomalous behaviour occurs at the critical acceleration, not at a distance, so it is difficult to explain the anomalies with dark matter. Since dark matter cannot be applied to them at these small scales, wide binary systems allow a purer comparison between competing theories of motion and, as shown here, quantised inertia predicts their behaviour better than MoND, without needing a tunable parameter (unlike MoND). As a caveat, it should be noted that other studies for example Banik (2019) claim that more wide binary data is needed to make the results of Hernandez (2019) conclusive.

\section{Method: Newtonian/GR}

Consider two stars, each one of mass $M$, mutually bound and orbiting. Applying Newton's second and gravity laws to one of the stars, we get

$F=M_{\mathrm{I}} a=\frac{G M_{\mathrm{g}} M_{\mathrm{g}}}{d^{2}}$

where $G$ is the gravitational constant, $d$ is the separation between the stars, $M_{\mathrm{I}}$ is the inertial mass of one star and $M_{\mathrm{g}}$ is its gravitational mass.

For a stable orbit gravity must be balanced by the centrifugal, inertial force. Assuming simple circular motion, so that $a=v^{2} / r=v^{2} /(d / 2)=2 v^{2} / d$, we get

$\frac{G M_{\mathrm{g}} M_{\mathrm{g}}}{d^{2}}=\frac{2 M_{\mathrm{I}} v^{2}}{d}$

where $v$ is the orbital velocity of each star. In standard physics, it is assumed that $M_{\mathrm{g}}=M_{\mathrm{I}}(\equiv M)$, and this gives the Newtonian orbital velocity:

$v_{\mathrm{N}}=\sqrt{\frac{G M}{2 d}}$ and finally we compute the relative velocity of the binary $\operatorname{system}(\Delta v=2 v)$ :

$\Delta v_{\mathrm{N}}=\sqrt{\frac{2 G M}{d}}$

Since the speeds involved (around $400 \mathrm{~m} / \mathrm{s}$ ) are low relative to the speed of light and gravitational forces are weak, the predictions of general relativity (GR) are indistinguishable from Newtonian dynamics for these cases.

\section{Method: MoND}

MoND assumes that for very low accelerations, either the strength of gravity or inertial mass is modified, see Milgrom (1983). Using inertial-MoND, Newton's second and gravity laws, replacing the inertial mass using the simple MoND function, Gentile et al. (2011), and naming $M_{\mathrm{g}}$ as $M$, we get

$F=M_{\mathrm{I}} a=\left(\frac{1}{1+\frac{a_{0}}{a+a_{\mathrm{g}}}}\right) M a=\frac{G M M}{d^{2}}$

where we have added the external field effect assuming the acceleration around the galaxy is, $a_{\mathrm{g}} \approx 1 \times 10^{-10} \mathrm{~m} / \mathrm{s}^{2}$, taking the lowest case from the Solar-system values determined by Iorio (2014) and Blanchet and Novak (2011) (all the binaries used here are within $100 \mathrm{pc}$ of the Sun, much smaller than the Sun's Galactocentric radius of $8.2 \mathrm{kpc}$ ). So that, after some algebra:

$a^{2}+\left(a_{\mathrm{g}}-\frac{G M}{d^{2}}\right) a-\frac{G M\left(a_{\mathrm{g}}+a_{0}\right)}{d^{2}}=0$

Applying $a=2 v^{2} / d$, we get

$v^{4}+\left(a_{\mathrm{g}} d-\frac{G M}{d}\right) \frac{v^{2}}{2}-\frac{G M}{4}\left(a_{\mathrm{g}}+a_{0}\right)=0$

Using the quadratic equation (and keeping just real and positive values) we get

$\Delta v_{\mathrm{MoND}}=\sqrt{\frac{G M}{d}-a_{\mathrm{g}} d+\sqrt{\left(a_{\mathrm{g}} d-\frac{G M}{d}\right)^{2}+4 G M\left(a_{\mathrm{g}}+a_{0}\right)}}$

\section{Method: quantised inertia}

Now, starting as before with Newton's second law and gravity law, replacing the inertial mass using quantised inertia (McCulloch 2007, see Eq. (1), above) and naming $M_{\mathrm{g}}$ as $M$ gives

$F=M_{\mathrm{I}} a=M\left(1-\frac{2 c^{2}}{A \Theta}\right) \frac{2 v^{2}}{d}=\frac{G M M}{d^{2}}$ 
where $A$ is the modulus of the total acceleration of the orbiting stars relative to the fixed stars, which includes $a=2 v^{2} / d$ which is just the radial centrifugal component plus the noncentrifugal acceleration of $2 c^{2} / \Theta$. Therefore

$\frac{G M}{2 d}=v^{2}-\frac{2 c^{2} v^{2}}{\left(\frac{2 v^{2}}{d}+\frac{2 c^{2}}{\Theta}\right) \Theta}$

Rearranging this, gives

$v^{4}-\frac{G M}{2 d} v^{2}-\frac{G M c^{2}}{2 \Theta}=0$

Using the quadratic formula (and keeping just real and positive values), the relative velocity predicted by quantised inertia is

$\Delta v_{\mathrm{QI}}=\sqrt{\frac{G M}{d}+\sqrt{\frac{G^{2} M^{2}}{d^{2}}+\frac{8 G M c^{2}}{\Theta}}}$

This formula has the advantage over the MoND formula in that it includes $2 c^{2} / \Theta$ instead of $a_{0}$ (the adjustable MoND parameter), meaning that all the parameters in Eq. (13) are fixed and known, so it cannot be tuned to the data. It either works or it does not, whereas Eq. (9) MoND has an arbitrary, tunable, parameter.

\section{Results}

The values input into these equations are as follows. An average solar mass in the study of Hernandez (2012) is $M=1 \times 10^{30} \mathrm{~kg}$, and in Eq. (9) the MoND fitting factor is set to its original value $a_{0}=1.2 \times 10^{-10} \mathrm{~m} / \mathrm{s}^{2}$, although it is true that some newer versions of MoND use a different value $\left(a_{0}=2 \times 10^{-10} \mathrm{~m} / \mathrm{s}^{2}\right)$, which gives an expression practically equivalent to QI, Eq. (13). The co-moving cosmic horizon used in Eq. (13) is $\Theta=8.8 \times 10^{26} \mathrm{~m}$. For all the models we assumed an uncertainty in mass ranging from $0.5 M$ to $2 M$, for MoND an uncertainty in $a_{0}$ ranging from 1.0 to $2.0 \times 10^{-10} \mathrm{~m} / \mathrm{s}^{2}$ and for QI an uncertainty in $\Theta$ of $9 \%$.

Figure 1 shows the separation in parsecs along the $x$ axis from 0.007 to $4 \mathrm{pc}$ and the relative orbital velocity of the orbiting binaries along the $y$ axis in $\mathrm{km} / \mathrm{s}$. The crosses show the rms relative velocity of the 83 wide binaries from the GAIA DR2 database as presented in Fig. 5 of Hernandez (2019). The grey area shows the uncertainty in these results. The five bins from left to right contained 21, 24, 17, 8 and 13 pairs of stars respectively, so that the narrower vertical error bars for the fourth bin is unexpected. As a check on our results for Newton and MoND, they are roughly in line with those from the detailed study of Banik and Zhao (2018)

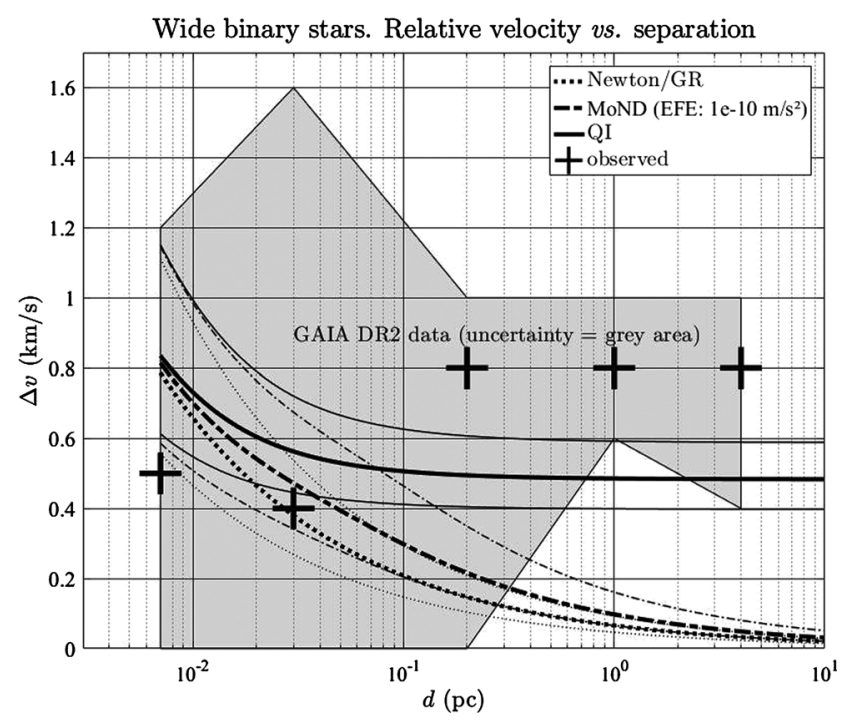

Fig. 1 The sky-projected relative velocities of the wide binaries from Hernandez (2019). The $x$-axis shows the separation (in pc), and the $y$ axis shows the relative velocity $(\mathrm{km} / \mathrm{s})$. The crosses and the grey areas show the observed velocities and possible range. The three dotted curves show the predicted Newtonian or general relativistic relative velocity and its upper and lower uncertainty bounds. The three dot-dashed curves are the predictions from MoND and its uncertainty bounds. The three solid curves represent the prediction of quantised inertia (and its uncertainty bounds). QI is the only model which agrees with the data

who found that at $20 \mathrm{kAU}$, or $0.1 \mathrm{pc}$, the prediction of Newton was $0.15 \mathrm{~km} / \mathrm{s}$ (compared to $0.2 \mathrm{~km} / \mathrm{s}$ here) and for MoND it was $0.2 \mathrm{~km} / \mathrm{s}$ (compared to $0.3 \mathrm{~km} / \mathrm{s}$ here). The difference in values could be due to the difference in assumed mass. They assumed the total mass to be $1.5 M_{\odot}$, we assumed $2 M_{\odot}$.

The dotted lines show the expected Newtonian or general relativistic velocity curve and its upper and lower uncertainty bounds. Newton/GR significantly underpredicts the observed speed for separations greater than about $0.3 \mathrm{pc}$ (60000 AU). So here general relativity is falsified and dark matter cannot be used to save it.

The dashed lines show the prediction of MoND and its upper and lower uncertainty bounds with the standard fitting parameter of $a_{0}=1.2 \times 10^{-10} \mathrm{~m} / \mathrm{s}^{2}$. This model is very similar to GR and also underpredicts the data for separations greater than $0.3 \mathrm{pc}$. This is due to the External Field Effect of MoND which means that the binary stars still have a large acceleration because of their orbit around the galactic centre, much higher than that needed to show significant MoND effects.

The solid lines show the prediction of quantised inertia and its upper and lower uncertainty bounds. QI alone agrees with the data given the error bars. The advantage of QI here is that it has no External Field Effect: the acceleration of the two stars relative to the galaxy does not affect the inertial mass used in calculations of their acceleration relative to 
each other. The other advantage of quantised inertia is that it requires no tuning parameter, $a_{0}$. It predicts this parameter itself. The data points all lie above the prediction of QI, but still in agreement. It is possible that some of the data has been contaminated by false binaries, though Hernandez (2019) took great care to avoid this. Nonetheless, Pittordis and Sutherland (2019) showed that false binaries are likely still an issue since a significant fraction of systems have relative velocities too high to be plausible in any gravity theory. These systems are likely to skew measures of wide binary self-gravity based on rms relative velocities.

\section{Discussion}

The following is a more intuitive discussion of the result. The binary stars' inertia is assumed in quantised inertia to be caused by Unruh waves that are produced as the two stars accelerate relative to other matter. This is due to the co-orbit of the two stars. As more widely-separated binary stars are considered, their acceleration relative to each other and to the rest of the matter in the universe decreases, with the proviso that it remains above $2 c^{2} / \Theta$. Therefore the Unruh waves, that are assumed in quantised inertia to determine their inertial mass, become longer, and a greater proportion of them are disallowed by the cosmic horizon (a Hubblescale Casimir effect). This means that the inertial mass of the widely separated stars decreases in a new way, and so they are able to orbit more quickly than expected, without the centrifugal (inertial) forces separating them (they remain bound).

As shown also by Hernandez (2012, 2019), these data are in tension with Newtonian or general relativity, since dark matter cannot be added to these systems, as it must stay spread out at this scale to fit galaxy rotations. MoND also does not predict these systems since the External Field Effect makes it equivalent to GR. Only QI agrees with the observed orbits within the uncertainty, and QI does it without needing any tuning, which is a significant advantage.

\section{Conclusion}

Wide binary data from GAIA DR2 disagree with general relativity since dark matter cannot be used in these cases.

MoND is also in disagreement with the data since the External Field Effect makes its predictions similar to those of general relativity.

Only quantised inertia (QI) agrees with the wide binary data, and QI also has the advantage that it needs no fitting parameter.
Acknowledgements Many thanks to the anonymous reviewers for their helpful comments, and to DARPA grant HR001118C0125.

Publisher's Note Springer Nature remains neutral with regard to jurisdictional claims in published maps and institutional affiliations.

Open Access This article is distributed under the terms of the Creative Commons Attribution 4.0 International License (http://creative commons.org/licenses/by/4.0/), which permits unrestricted use, distribution, and reproduction in any medium, provided you give appropriate credit to the original author(s) and the source, provide a link to the Creative Commons license, and indicate if changes were made.

\section{References}

Ahmed, Z., et al. (CDMS Collaboration): Phys. Rev. Lett. 102, 011301 (2009)

Banik, I.: A new line on the wide binary test of gravity. Mon. Not. R. Astron. Soc. 487, 5291-5303 (2019)

Banik, I., Zhao, H-S.: Testing gravity with wide binary stars like $\alpha$ Centauri. Mon. Not. R. Astron. Soc. 480(2), 2660-2688 (2018)

Bars, I., Terning, J.: Extra Dimensions in Space and Time. Springer, Berlin (2009)

Blanchet, L., Novak, J.: External field effect of modified Newtonian dynamics in the Solar system. Mon. Not. R. Astron. Soc. 412(4), 2530-2542 (2011)

Gentile, G., Famaey, B., de Blok, W.J.G.: THINGS about MoND. Astron. Astrophys. 527, A76 (2011)

Hernandez, X.: Eur. Phys. J. C 72(2), 1884 (2012)

Hernandez, X.: Int. J. Mod. Phys. D 28(8), 1950101 (2019)

Iorio, L.: On the MOND external field effect in the Solar system. Astrophys. Space Sci. 323(3), 215-219 (2014)

McCulloch, M.E.: Modelling the Pioneer anomaly as modified inertia. Mon. Not. R. Astron. Soc. 376, 338-342 (2007)

McCulloch, M.E.: Testing quantised inertia on galactic scales. Astrophys. Space Sci. 342(2), 575-578 (2012)

McCulloch, M.E.: Inertia from an asymmetric Casimir effect. Europhys. Lett. 101, 59001 (2013)

McCulloch, M.E.: Quantised inertia from relativity and the uncertainty principle. Europhys. Lett. 115, 69001 (2016)

McCulloch, M.E.: Galaxy rotations from quantised inertia and visible matter only. Astrophys. Space Sci. 362, 149 (2017a)

McCulloch, M.E.: Testing quantised inertia on emdrives with dielectrics. Europhys. Lett. 118, 34003 (2017b)

Milgrom, M.: A modification of the Newtonian dynamics as a possible alternative to the hidden mass hypothesis. Astrophys. J. 270, 365 (1983)

Pittordis, C., Sutherland, W.: Testing modified gravity with wide binaries in GAIA DR2. Mon. Not. R. Astron. Soc. (2019). https:// doi.org/10.1093/mnras/stz1898

Rubin, V.C., Ford, W.K. Jr., Thonnard, N.: Rotational properties of $21 \mathrm{Sc}$ Galaxies with a large range of luminosities and radii, from NGC $4605(R=4 \mathrm{kpc})$ to UGC $2885(R=122 \mathrm{kpc})$. Astrophys. J. 238, 471 (1980)

Sanders, R.H., McGaugh, S.S.: Annu. Rev. Astron. Astrophys. 40, 263 (2002)

Scarpa, R., Marconi, G., Gimuzzi, R., Carraro, G.: Astron. Astrophys. 462, L9 (2007)

XENON10 Collaboration: Phys. Rev. D 80, 115005 (2010)

Zwicky, F.: Der Rotverschiebung von extragalaktischen Nebeln. Helv. Phys. Acta 6, 110 (1933) 